\title{
Measuring the Core Shift EfFect in AGN Jets WITH THE EXTENDED KOREAN VLBI NETWORK
}

\author{
Taehyun Jung ${ }^{1,2}$, Richard Dodson ${ }^{3}$, Seog-Tae Han $^{1}$, MAría J. Rioja $^{3,4}$, Do- Young Byun ${ }^{1}$, Mareki Honma $^{5}$, \\ Jamie Stevens ${ }^{6}$, Pablo de Vicente ${ }^{4}$, And Bong Won Sohn ${ }^{1,2}$ \\ ${ }^{1}$ Korea Astronomy and Space Science Institute, 776 Daedeokdae-ro, Yuseong-gu, Daejeon 305348, Korea \\ ${ }^{2}$ University of Science and Technology, 217 Gajeong-ro, Yuseong-gu, Daejeon 305350, Korea \\ ${ }^{3}$ International Centre for Radio Astronomy Research, M468, The University of Western Australia, 35 Stirling Hwy, \\ Crawley, Western Australia 6009, Australia \\ ${ }^{4}$ Observatorio Astronómico Nacional (IGN), Alfonso XII, 3 y 5, 28014 Madrid, Spain \\ ${ }^{5}$ Mizsawa VLBI Observatory, National Astronomical Observatory of Japan, Japan \\ ${ }^{6}$ Australia Telescope National Facility, CSIRO, Australia \\ Received September 2, 2015; accepted October 15, 2015
}

\begin{abstract}
We present our efforts for extending the simultaneous multi-frequency receiver system of the Korean Very Long Baseline Interferometry (VLBI) Network (KVN) to global baselines in order to measure the frequency-dependent position shifts in Active Galactic Nuclei (AGN) jets, the so called core shift effect, with an unprecedented accuracy (a few micro-arcseconds). Millimeter VLBI observations with simultaneous multi-frequency receiver systems, like those of the KVN, enable us to explore the innermost regions of AGN and high precision astrometry. Such a system is capable of locating the frequency dependent opacity changes accurately. We have conducted the feasibility test-observations with the interested partners by implementing the KVN-compatible systems. Here we describe the science case for measuring the core shift effect in the AGN jet and report progress and future plans on extending the simultaneous multi-frequency system to global baselines.
\end{abstract}

Key words: techniques: interferometeric — astrometry — high angular resolution — radio continuum: active galactic nuclei

\section{INTRODUCTION}

Very Long Baseline Interferometry (VLBI) at (sub)mm wavelengths results in the highest angular resolutions achieved in astronomy and has a unique access to emission regions that are inaccessible with any other approach or at longer wavelengths, because the extremely compact areas of interest are often selfabsorbed. Therefore, it holds a unique potential for increasing our understanding of the physical processes in e.g., Active Galactic Nuclei (AGN) and in the vicinity of supermassive black holes.

Continuous development and technical improvements have led to a sustained increase of the high frequency threshold for VLBI observations in the last two decades (Krichbaum et al. 2014). Regular observations up to $86 \mathrm{GHz}$ are being carried out with well-established networks, such as the Very Long Baseline Arrary (VLBA) and Global Millimeter VLBI Array (GMVA), more recently up to $140 \mathrm{GHz}$ with the Korean VLBI Network (KVN) and ad-hoc observations at the highest frequencies up to $240 \mathrm{GHz}$ (Doeleman et al. 2008). The field of mm-VLBI will greatly benefit from the arrival of phased-up Atacama Large Millimeter/submillimeter Array (ALMA) (Matthews \& Crew 2015) for joint VLBI observations.

VLBI studies at centimeter wavelengths are a well-

CORRESPONDING AUTHOR: T. Jung established field, with advanced technological developments and analysis techniques that result in superb quality images, including those of very weak $\mu \mathrm{Jy}$ sources (Garrett 2005; Chi et al. 2013) and with microarcsecond ( $\mu$ as) astrometry (Reid \& Honma 2014), using phase referencing techniques. However, this type of study in mm-VLBI is much less widespread because the observations become progressively more challenging as the wavelength gets shorter. This is because of the limited telescope surface accuracy and efficiency, receiver system temperatures and sensitivity, shorter atmospheric coherence times, and because sources are intrinsically weaker in general. Therefore, phase referencing techniques that are routine in cm-VLBI fail to work beyond $43 \mathrm{GHz}$, excluding a single case at $86 \mathrm{GHz}$ with the VLBA (Porcas \& Rioja 2002).

For AGN studies, the detection of the point of the transition from optically thick to optically thin emission as a function of frequency is a field of intense investigation, for example, an extensive survey of the core shift effect in AGN jets (Sokolovsky et al. 2011) in the centimeter wavelengths and a beautiful demonstration on M87 (Hada et al. 2011).

Furthermore maps of the spectral index or rotation measure across the source, at mm-wavelengths, provide crucial insights into the development of the magnetic field strength and particle densities, as the jet exits the core region and extends down the outflow. However, 


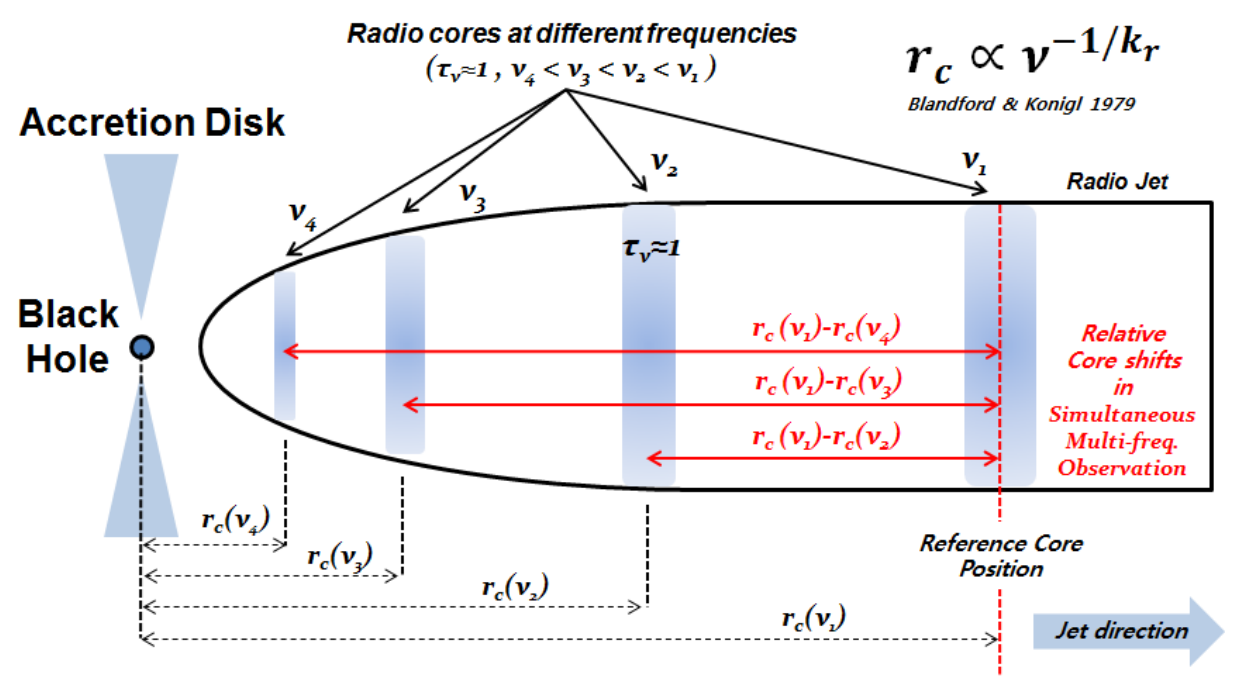

Figure 1. A new approach for measuring the frequency-dependent VLBI core position shifts in AGN jet with the simultaneous multi-frequency receiver system. The relative position of cores at higher frequencies $\left(\nu_{2}, \nu_{3}, \nu_{4}\right)$ is measured by referencing the core position at the lower frequency $\left(\nu_{1}\right)$. This figure is modified from Hada et al. (2011).

astrometric registration is important to form meaningful interpretation. There are a number of methods in AGN studies which can be used to align images between frequencies, as discussed in Hovatta et al. (2014). They argued that any results derived without accurate phase referencing are questionable close to the core, which are the regions most of interest in mm-VLBI.

Jung et al. (2011) demonstrated the excellence of multi-frequency phase referencing on atmospheric phase compensation in mm-VLBI. Based on this advantage, the KVN (Kim et al. 2004; Lee et al. 2014) as the first dedicated mm-VLBI array, addresses one of the fundamental limitations of the field, the atmospheric stability, by introducing the simultaneous multi-frequency receiver system (Han et al. 2008). The KVN currently consists of three antennas spread across South Korea, located in Seoul and Ulsan in the main land, and in Jeju island. The observing frequencies are $22,43,86$ and $129 \mathrm{GHz}$. The baseline lengths between the antennas range between 300 and $500 \mathrm{~km}$, which provides a spatial resolution $\sim 1$ mas at the highest frequency band.

However, the KVN system is limited to the three antennas on the South Korean Peninsula, which have very short baselines and, therefore, a very large synthesized beam size even though it can perform up to $140 \mathrm{GHz}$ VLBI observations. To facilitate higher resolutions, we require long baselines. In order to maximize the observing capability at millimeter wavelengths, these long baselines must have the same capacity as the KVN for the same calibration strategies to be applied. Therefore, we have been seeking out partners for the KVN who will be able to co-observe simultaneously at several different frequencies. This is the extended-KVN (x-KVN) project and has been funded under the pilot project of 'Challenging the Future' by Korean Astronomy and Space Science Institute (KASI). The key science in this project is to measure the core shifts directly
Table 1

Expected angular resolution and astrometric accuracy.

\begin{tabular}{cccccc}
\hline \multirow{2}{*}{$\begin{array}{c}\text { Frequency } \\
(\mathrm{GHz})\end{array}$} & \multicolumn{2}{c}{ Resolution $(\mathrm{mas})$} & & \multicolumn{2}{c}{ Accuracy $(\mu \mathrm{as})$} \\
\cline { 2 - 3 } \cline { 5 - 6 } & KVN & x-KVN & & KVN & x-KVN \\
\hline 22 & 6 & 0.33 & & 346 & 12 \\
43 & 3 & 0.16 & & 173 & 6 \\
86 & 1.5 & 0.08 & & 87 & 3 \\
129 & 1.0 & 0.05 & & 58 & 2 \\
\hline
\end{tabular}

The angular resolution (Column 2) of the three antennas of the KVN at the supported frequencies and that of a proposed extended KVN (x-KVN, Column 3) with a maximum baseline of $9,000 \mathrm{~km}$ (the number of antennas $\left(\mathrm{N}_{\text {ant }}\right)$ being 8 ), in mas. With a per-baseline SNR of 10 , we would expect to have astrometric accuracies of the order of the resolution/SNR $/ \sqrt{N_{\text {ant }}}$, which are given in $\mu$ as in Columns 4 and 5 for KVN and $\mathrm{x}-\mathrm{KVN}$, respectively.

at multiple frequencies by the $\mathrm{x}-\mathrm{KVN}$. The details are described in the next Section. The international partners are listed in Section 3. We summarized the technical options for the simultaneous multi-frequency receiver system in Section 4, and its demonstration is presented in Section 5. Summary and future plans are given in Section 6.

\section{Measuring the Core Shift Effect WITH GLOBAL BASELINES}

According to Blandford \& Königl (1979) the position of VLBI cores, $r_{\text {core }}$, where the most of emission comes from (optical depth, $\tau \sim 1$ ) at a given observing frequency $\left(\nu_{o b s}\right)$, varies with distance from the central engine. This frequency-dependent position shift in AGN jets is called the 'core shift effect' and is described as $r_{\text {core }}(\nu) \propto \nu_{\text {obs }}^{-1 / k}$, where $k$ is a coefficient related to the magnetic field, particle density distribution, and the shape of the electron energy distribution. The core position measured at centimeter wavelengths with respect 


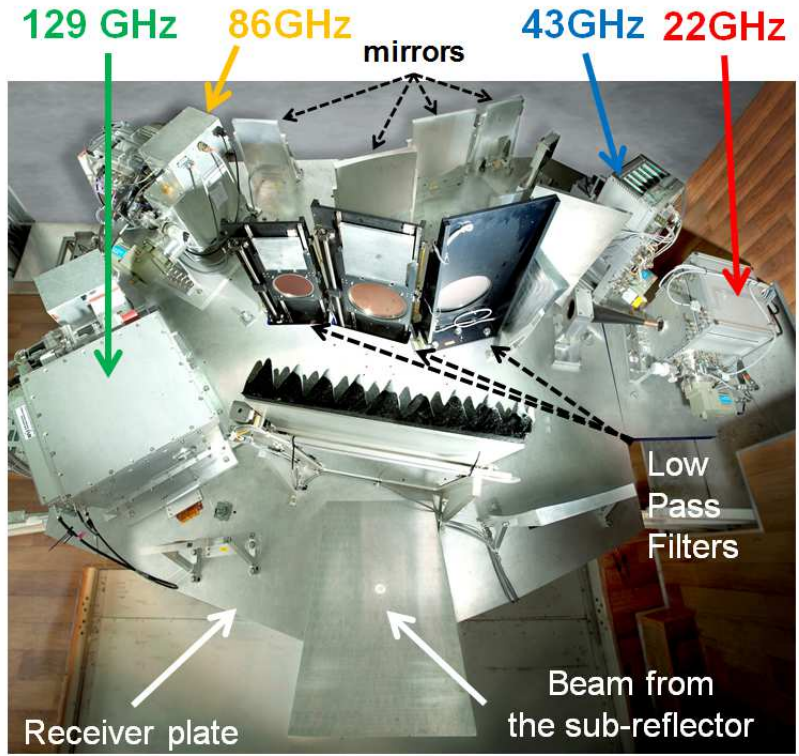

Figure 2. Top view of the simultaneous multi-frequency receiver system installed at the receiver cabin of KVN Yonsei antenna. The 4-frequency receivers (22, 43, 86, and 129 $\mathrm{GHz}$ ) are installed on the receiver plate with quasi-optical mirrors and three low pass filters, which divide the signal from the subreflector to the appropriate receiver (Han et al. 2013).

to an optical thin jet component are consistent with $r_{\text {core }}(\nu) \propto \nu_{\text {obs }}^{-1}$, interpreting the core as a conical jet in equipartition exhibiting synchrotron self-absorption (Sokolovsky et al. 2011).

However, a study of the core shift effect in the innermost area of AGN, very close to the central black hole and jet-launching region, has been prohibited because of the observational difficulties. Only high frequency VLBI observations with high angular resolution are able to access this region due to the opacity effect. In addition, high precision astrometric capability is essential to measure the core shift effect accurately, since the variation of core position at high frequencies will be much less than that of at low frequencies (e.g., $\propto \nu_{\text {obs }}^{-1}$ ).

Core shifts in VLBI are usually measured by aligning the optically thin features (superluminar feature where $\tau<1$ ) as a reference point to the cores at each frequency (Kovalev et al. 2008; Sokolovsky et al. 2011). With the simultaneous multi-frequency receiver system, however, the relative position of cores at different frequencies is measured by referencing the core position at the lower frequency, since the lower frequency phase is used as a phase reference for astrometry of high frequency data and its calibration. This new approach is illustrated in Figure 1.

One can calculate the resolution that one will obtain with the KVN at the four frequency bands and compare these resolutions with the typical size of core shifts. Core shifts between 2.3 and $8.6 \mathrm{GHz}$ have a typical size of 0.44 mas for the 29 selected AGN in that study of Kovalev et al. (2008). More recent results with the

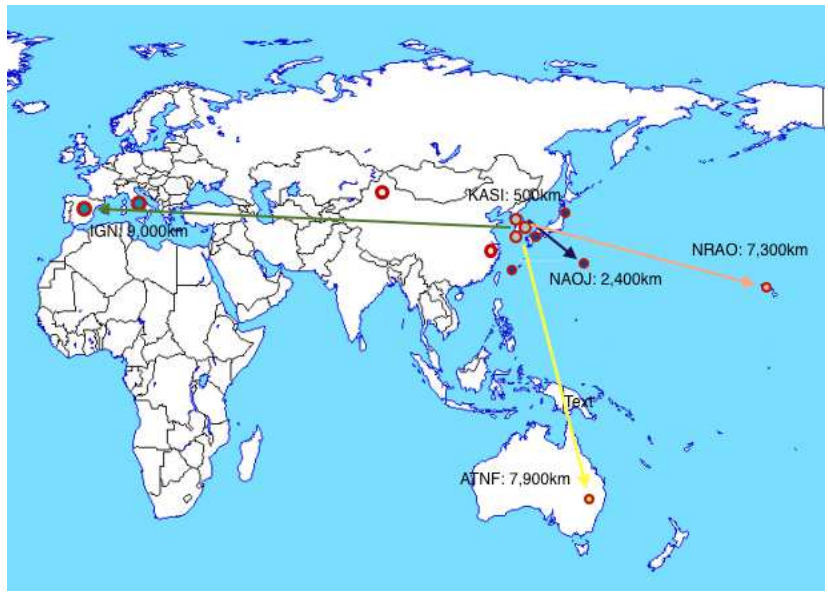

Figure 3. Partner institutions in the $x-K V N$ project. KASI manages the three KVN antennas that have baselines of $\sim 500 \mathrm{~km}$; NAOJ manages the four VERA antennas that have baselines of 2,300 km; CSIRO Astronomy and Space Science (CASS) manages the six ATCA antennas that provides a baseline of $7,900 \mathrm{~km}$; IGN manages the Yebes antenna that gives a baseline of $9,000 \mathrm{~km}$; NRAO manages the VLBA antennas including Mauna Kea with a baseline of $7,300 \mathrm{~km}$.

VLBA show typical core shift values of 1.2 mas between 1.4 and $15.4 \mathrm{GHz}$, and of 0.24 mas between 5 and 15.4 $\mathrm{GHz}$, indicating an $r_{\text {core }}(\nu) \propto \nu_{\text {obs }}^{-1}$ law (Sokolovsky et al. 2011).

Therefore, if they follow the standard Blandford \& Königl (1979) model, the core shifts at millimeter frequencies would be expected to be $\sim 50 \mu$ as between 22 and $129 \mathrm{GHz}$. Whereas the resolution, even at $129 \mathrm{GHz}$, is barely less than a mas and the astrometric accuracy only about twenty times better than that, for a perbaseline Signal to Noise Ratio (SNR) of $\sim 10$. Table 1 lists the resolution from the KVN at the four frequencies and the resolution and potential accuracy that could be obtained with 8-station global array with maximum baselines of 9,000 km (those between Europe and Korea). The resolutions on global baselines are matching the expected core shift scales with the astrometric accuracies twenty times better, demonstrating the importance of global baselines for meaningful core shift studies.

\section{Global Expansion of the Simultaneous MULTI-FREQUENCY RECEIVER SYSTEM}

\subsection{Simultaneous Multi-Frequency Receiver System in mm-VLBI Observation}

The non-dispersive characteristic of the neutral troposphere to the radio signals is well known. This provides an effective way to overcome difficulties due to the troposphere, in particular the highly variable wet components, by using the simultaneous multi-frequency receiver system. Therefore, one can calibrate the tropospheric fluctuations that is usually problematic at higher high frequency (e.g., 43, 86 and $129 \mathrm{GHz}$ in case of the $\mathrm{KVN}$ ) using the phase solutions obtained from 


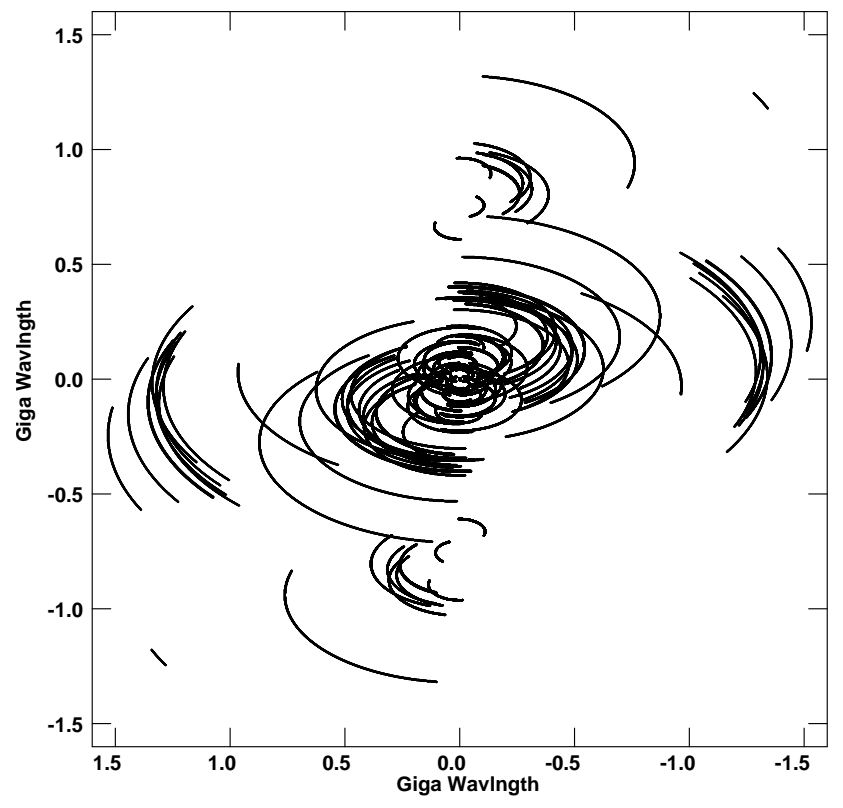

Figure 4. The simulated $u v$ coverage of global baselines for a source at $30^{\circ}$ in declination at $43 \mathrm{GHz}$ (reference antenna: KVN Tamna). Twelve antennas are considered: three KVN, four VERA, Shanghai 65-m, Urumqi, Yebes 40-m, ATCA and VLBA Mauna Kea. The inner part corresponds to KVN and VERA baselines, and the outer part comes from longer baselines of Yebes (east-west) and ATCA (north-south). The Urumqi (east-west) and VLBA Mauna Kea (northwest-southeast) baselines fill the middle part.

the lower frequency (e.g., 22 and/or $43 \mathrm{GHz}$ ). Jung et al. (2011) successfully demonstrated the excellence of the simultaneous dual-frequency observation on compensating the atmospheric phase fluctuations at 22 and $43 \mathrm{GHz}$ using the VLBI Exploration of Radio Astrometry (VERA) as a feasibility study for the simultaneous multi-frequency receiver system of the KVN.

The multi-frequency receiver system of KVN (Han et al. 2008, 2013) is designed for atmospheric compensation using simultaneous observations at multiple frequencies, which results in an effective increase of the coherence time, well beyond that imposed by atmospheric propagation (Figure 2) and its performance was proved for the first time by Jung (2011). The KVN backend, combined with the Source Frequency Phase Referencing (SFPR) data analysis technique (Dodson \& Rioja 2009; Rioja \& Dodson 2011; Rioja et al. 2011, 2014, 2015), allows high precision astrometric measurements even at the highest frequencies. We know of no demonstrated upper frequency limit and the method would be expected to work as long as the tropospheric propagation effects were non-dispersive. In this context, successful tests are on-going with ALMA (Fomalont et al. 2014) at frequencies as high as $650 \mathrm{GHz}$ (where this is known as the Band-to-Band (B2B) mode). In Rioja et al. (2015), we presented results of SFPR astrometric measurements with the KVN at all four frequency bands, for continuum sources.

\subsection{Interested Radio Astronomy Partners}

A significant number of radio astronomy institutions have expressed an interest in KVN-compatible VLBI systems. We have selected the most compatible partner institutions for our initial investigations, as shown in Figure 3. These are the National Astronomical Observatory of Japan (NAOJ) with their four 20-m VERA antennas close to Korea, the Australia Telescope National Facility (ATNF) with their six 22-m Australia Telescope Compact Array (ATCA) antennas in Narrabri, Australia, and the National Geographic Institute (IGN) with their 40-m antenna in Yebes, Spain. We also consider other parties for future expansion: the new Shanghai $65-\mathrm{m}$ and Urumqi $26-\mathrm{m}$ antennas in China, the Italian Sardinia 64-m antenna, and the National Radio Astronomy Observatory (NRAO), starting with one of their VLBA antennas in Mauna Kea. The expected $u v$ coverage of partners above at $43 \mathrm{GHz}$ for a source at $30^{\circ}$ in declination is presented in Figure 4, indicating that we would expect both high resolution and good image quality. In this example, the resolution which would be provided is $\sim 160 \mu$ as at $43 \mathrm{GHz}$ (Table 1).

\section{TEChNICAL OPTIONS TO PROVIDE KVN-COMPATIBLE VLBI BACKENDS}

\subsection{Fast Switching between Receivers}

This is the system which is in place on the VLBA (Napier 1995) and has been demonstrated to be suitable for compensating atmospheric phase fluctuations to detect weak sources at high frequency (Middelberg et al. 2005) and high frequency astrometry by SFPR (Dodson \& Rioja 2009; Rioja \& Dodson 2011). It has been proposed as a possible implementation on the Sardinia 64-m antenna, Italy. This is probably the simplest solution under consideration, but will always have an upper limit to the astrometric accuracy as there has to be an interpolation of solutions between frequencies, which are not observed simultaneously. It also has a low observing efficiency as a significant portion of the time is essentially off-source, as discussed in Rioja et al. (2014).

\subsection{Sub-Array Solution}

For a phased-up array of telescopes, one solution is to have different sub-arrays, observing the different frequency bands. Such a mode has been implemented on the ATCA in Australia. This requires the implementation of independent feed rotation for each antenna so that the correct receiver package could be placed on-axis, plus a number of minor features for the management of a sub-arraying operating mode. As the first local oscillator is common for all antennas, with different multipliers for the different bands, there are limited frequency ranges which are allowed in each of the configurations. Details of what can be supported are to be found on the ATNF website ${ }^{\mathbf{1}}$, but in summary, only the

\footnotetext{
$\mathbf{1}_{\text {WwW.narrabri.atnf.csiro.au/people/ste616/mm_simultaneous }}$
} 

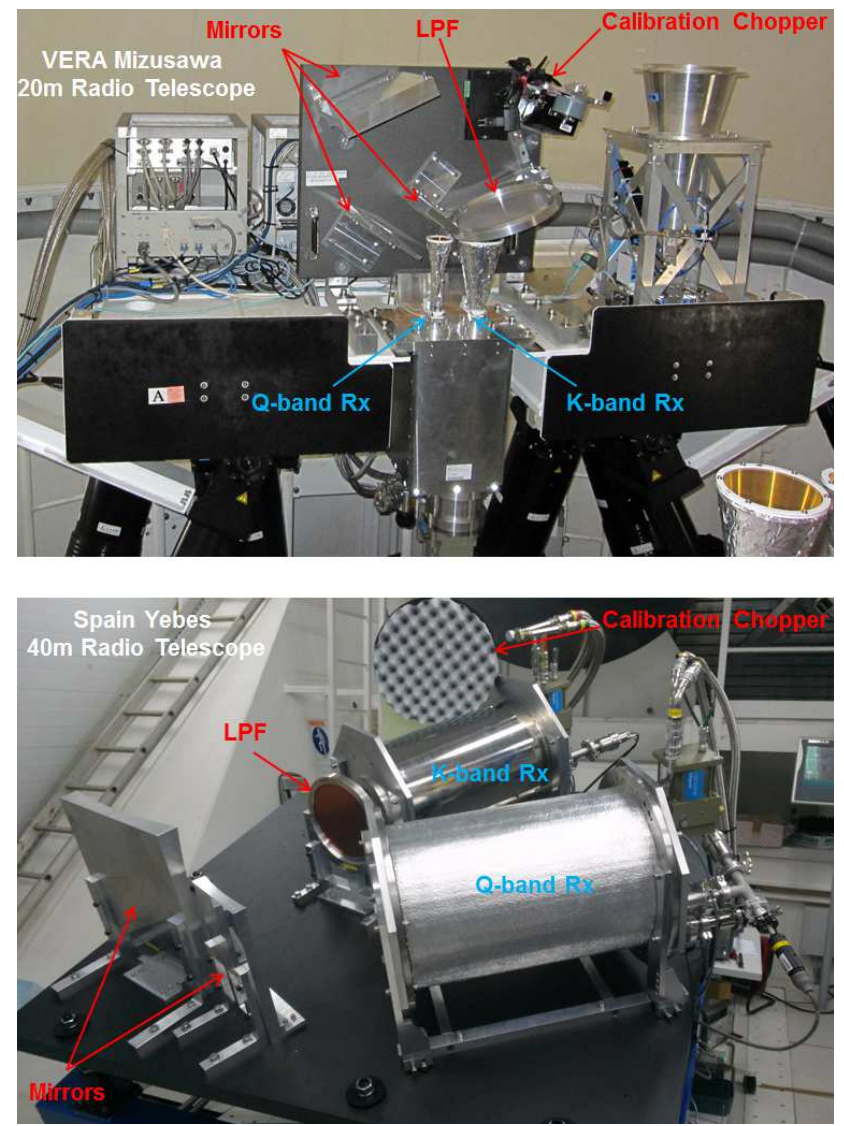

Figure 5. Photographs of K/Q-band quasi-optical system installed in global baselines. Top: VERA Mizusawa 20-m antenna in Japan. Bottom: Yebes 40-m antennas in Spain.

Q-band frequencies between 44.9 and $50 \mathrm{GHz}$ are available when observing $\mathrm{K}$ and $\mathrm{Q}$ bands simultaneously. No configuration allows for observing between $\mathrm{K}$ and $\mathrm{W}$ bands. When observing $\mathrm{Q}$ and $\mathrm{W}$ bands, W-band must lie between 86.6 and $102 \mathrm{GHz}$.

The challenge for ATCA is to maintain the phasing up of the array whilst subsections are observing at different frequencies. The current solution has only demonstrated short-term maintenance of the array coherence. In the future, we will swap regularly between the sub-array mode and normal mode, where we can re-phase the array. Alternative solutions without the same observing time overhead are being searched for.

\subsection{Quasi-Optical Receiver System}

There are a number of designs for traditional QuasiOptical (QO) systems that enable one to observe target sources at different frequencies simultaneously (see Goldsmith (1998) for a review of the field). This approach is extremely flexible and well studied, so there is a large pool of experience to draw on. However, the traditional QO systems have very low efficiency and have a large receiver cabin footprint. These two facts have weighed against the wide-spread implementation of the approach.

Designs for retro-fitting the KVN QO system into the existing infrastructure for several antennas have been developed at KASI, under the pilot project. The first demonstration of this is the installation on the VERA receiver platform (Mizusawa 20-m antenna) in 2014 (Figure 5). After the demonstration, two sets of QO systems have been produced and will be installed at Mizusawa and Iriki antennas of the VERA in October 2015.

Where it is possible to build a new platform without tight limitations of space, such as for the Yebes $40-\mathrm{m}$ radio-telescope that has a Nasmyth optical design providing a great amount of room in the receiver cabin, it is more straight-forward to provide a new feed platform where the receivers can be mounted. This was done for a demonstration at Yebes in 2014 (see Figure 5 for photograph of the installation). Although the KVN QO system is a huge advance on traditional QO systems, having very high efficiency while much smaller than traditional configurations, this still has a significant receiver cabin footprint.

\subsection{Tri-Band Feed System}

Dual-band feeds with concentric horns, are well established in radio astronomy. The geodetic SX receivers, which have the $8.4 \mathrm{GHz}$ feed inside the $2.4 \mathrm{GHz}$ feed, have been used for decades (Williams et al. 1979). Recently, the Centre for Technology and Design (Centro de Desarrollos y Tecnológicos, CDT), part of IGN, have presented a tri-band feed for $\mathrm{S}, \mathrm{X}$ and $\mathrm{Ku}$ band geodetic installations (López-Pérez et al. 2012).

A tri-band solution would be a very efficient solution of the requirements, being not significantly larger than a single pixel feed. In particular, this is an ideal system to minimize errors induced by a quasi-optical system: for example, the system noise increased by passing through quasi-optics and low pass filters. Despite the advantages of a tri-band system, the design requirements of the simultaneous $\mathrm{K}, \mathrm{Q}$ and $\mathrm{W}$-band receiver system might be hard to meet when the frequency ratio's are significantly smaller than those previously developed. We would require frequency ratio's of $\sim 2$ (e.g., tri-band frequency of 22,43 and $86 \mathrm{GHz})$, where the existing systems have ratio's of $\sim 3.5$ (e.g., tri-band frequency of 2,8 and $30 \mathrm{GHz}$ ).

\subsection{Triple Feed-Single Dewar System}

A hybrid solution which could meet all the requirements above would be to pack all the mm-receivers and the KVN optical system into one dewar. This is made possible by recent developments in producing very small feed horns for the lower frequencies on ALMA (Zorzi et al. 2012). It should be noted that these particular design investigations are at a very preliminary stage.

\section{Demonstrations}

\subsection{Demonstrations with the VERA Antennas}

The proto-type QO system was installed between the $\mathrm{K}$ and Q-band receivers of VERA Mizusawa antenna in 2014. The fringe test observation between KVN and 

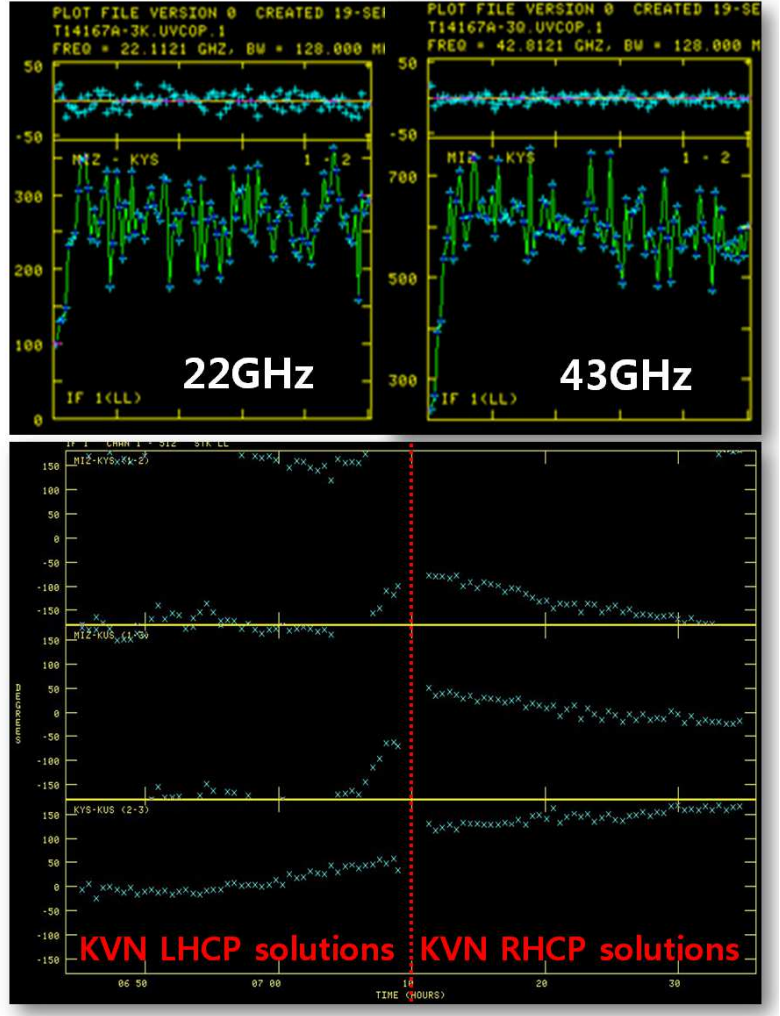

Figure 6. The first simultaneous VLBI fringes at 22 and 43 $\mathrm{GHz}$ between VERA and KVN, in June 2014. Top: The cross power spectra (fringes) of OJ287 at 22 and $43 \mathrm{GHz}$ at the Mizusawa and Yonsei baseline. Bottom: The visibility phase of OJ287 at $43 \mathrm{GHz}$, calibrated by using the $22 \mathrm{GHz}$ phase solutions among three baselines of Mizusawa (MIZ), Yonsei (KYS) and Ulsan (KUS) telescopes: (top) MIZ-KYS, (mid) MIZ-KUS and (bottom) KYS-KUS.

VERA was performed in June 2014, and the first simultaneous VLBI fringes at 22 and $43 \mathrm{GHz}$ were successfully obtained (Figure 6). The visibility phases of OJ287 at $43 \mathrm{GHz}$, calibrated by using the $22 \mathrm{GHz}$ phase solutions show a smooth variation during $\sim 50$ minutes experiment, improving the phase coherence to more than 20 minutes at $43 \mathrm{GHz}$. These results imply that the QO system can compensate for the atmospheric phase fluctuations effectively, and thus are very promising for implementing the simultaneous multi-frequency receiver system to other existing VLBI antennas.

\subsection{Demonstrations with the ATCA Antennas}

Several demonstrations of observations between KVN and ATCA have been run in 2014-2015. These have allowed us to shake down the system and identify the issues. Tests have focused on the simultaneous observations of the $\mathrm{Q}$ and $\mathrm{W}$ bands, as there currently is no overlap in the frequency span of ATCA and KVN at Q-band when also observing at K-band. The first image of M87 at W-band ( $87 \mathrm{GHz}$ ) between the KVN and ATCA baselines was obtained in 2015 (Figure 7). Its phase at W-band was calibrated by using the Q-band (43 GHz) phase solutions. Several important techni-

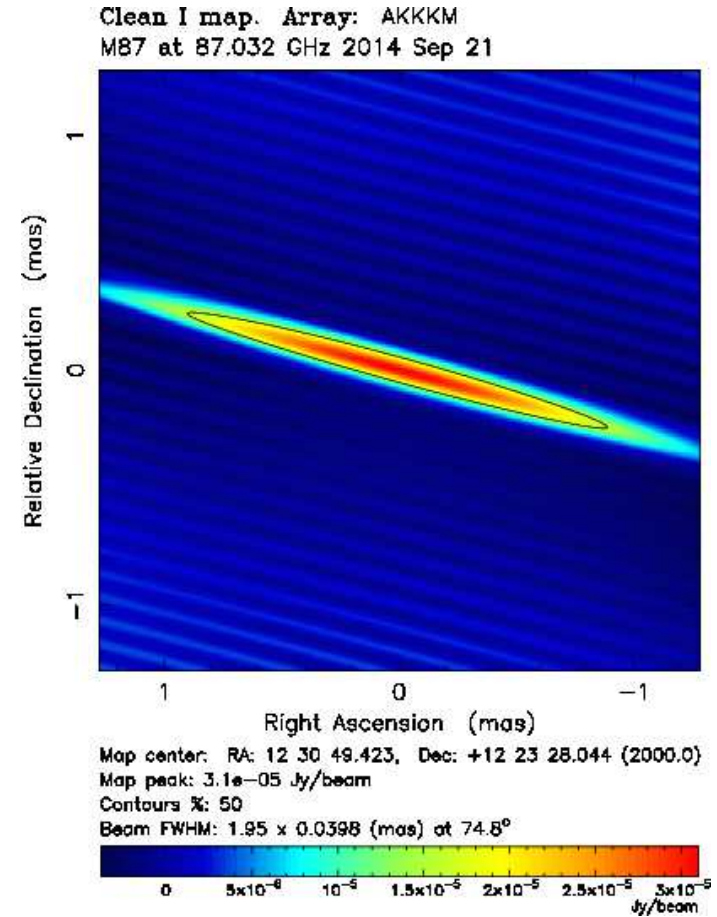

Figure 7. The first image of M87 at W-band $(87 \mathrm{GHz})$ of which phase is calibrated by using the Q-band (43 GHz) phase solutions between the ATCA and KVN baselines in 2015. The image is produced by further phase selfcalibration, but no amplitude calibration is applied.

cal issues need to be resolved, in particular on the best strategy to maintain the array coherence.

\subsection{Demonstrations with the Yebes Antenna}

The proto-type QO system was installed on the Yebes 40- $\mathrm{m}$ antenna in January 2015 (Figure 5). This is configured for simultaneous observing of K, Q and W-band, but only the first two are installed at present. Test observations between Yebes and KVN were performed in April 2015, and simultaneous fringes at 22 and 42 $\mathrm{GHz}$ were successfully obtained, as shown in Figure 8. We also confirmed dual-polarization fringes using the 1 Gbps recording mode (four IF channels with $32 \mathrm{MHz}$ for each polarization).

\section{Summary and future Plans}

In this work, we present our efforts for extending the simultaneous multi-frequency receiver system of KVN to global baselines in order to measure the core shift effect with an unprecedented accuracy (a few $\mu$ as). Measuring the effect of frequency-dependent core shift of AGN jets using VLBI provides a unique tool to study conditions at the central regions of AGN. In particular, mm-VLBI observations with the simultaneous multifrequency receiver system have significant benefits such as: i) unparalleled access to the innermost region of AGN, where the supermassive black hole resides and the jet is launched, ii) providing constraints on jet conditions that can be changed over the observation period 

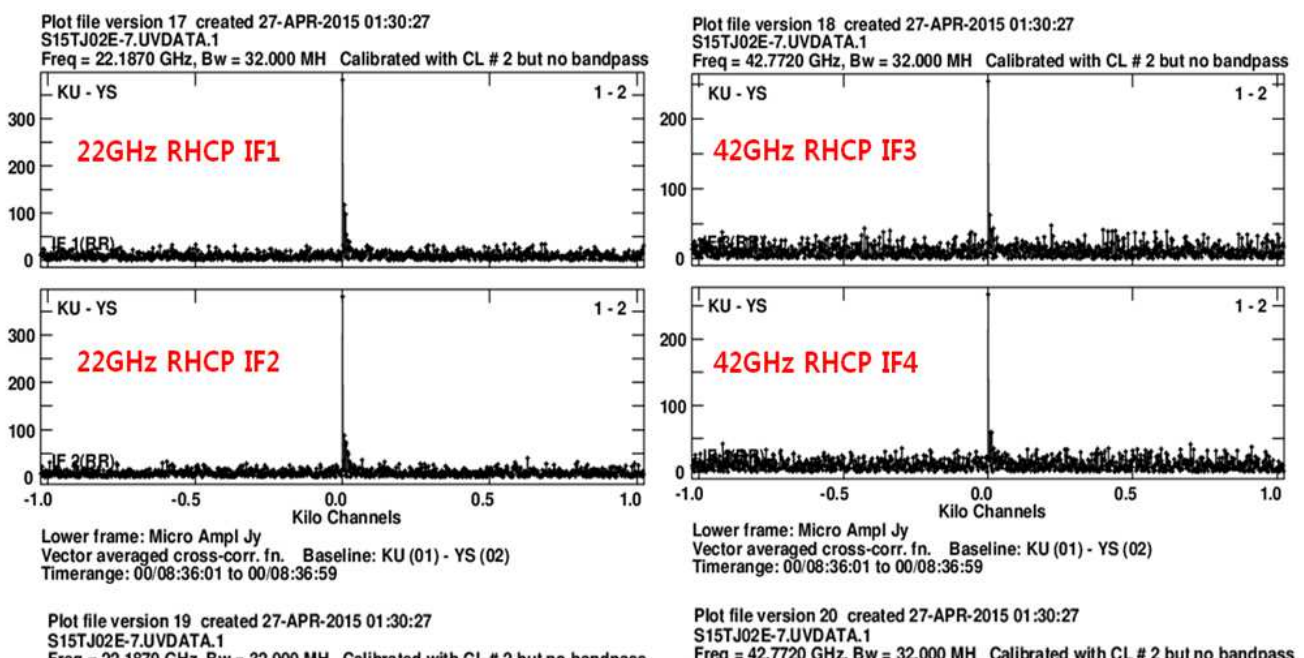

Vector averaged cross-corr. fn. Baseline: KU (01) - YS (02)
Timerange: 00/08:36:01 to $0008: 36: 59$

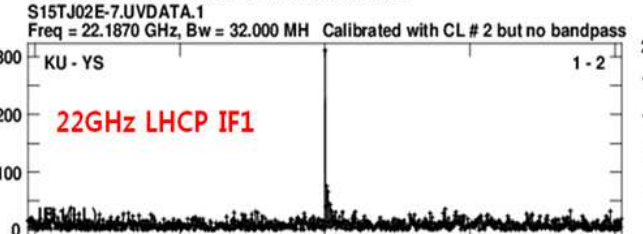

Plot file version 20 created 27-APR-2015 01:30:27

Freq $=42.7720 \mathrm{GHz}, \mathrm{BW}=32.000 \mathrm{MH}$ Calibrated with $\mathrm{CL} \# 2$ but no bandpass
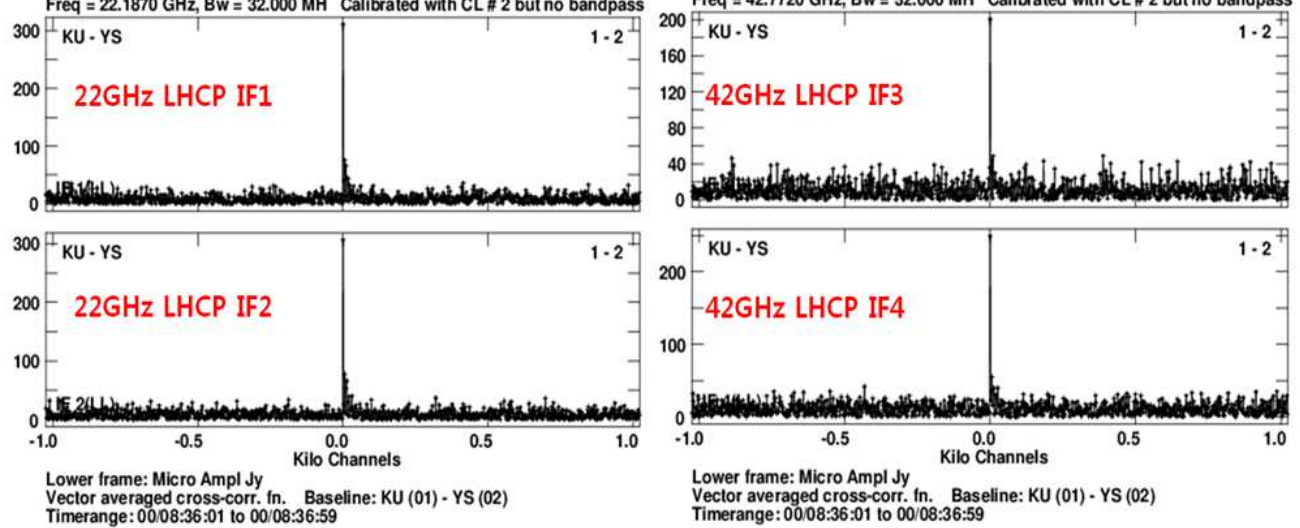

Figure 8. The first simultaneous VLBI fringes at 22 and $42 \mathrm{GHz}$ between Yebes $40-\mathrm{m}$ and KVN Ulsan antennas in April 2015. The observation was made using the full polarization mode at 1 Gbps data rate. Four IF channels with $32 \mathrm{MHz}$ subbands for each polarization was configured. Upper and lower plots correspond to RHCP and LHCP, while right and left plots represent the simultaneous fringes at 22 and $42 \mathrm{GHz}$, respectively.

at different frequencies, and iii) high precision astrometry resulting in perfect tropospheric error calibration.

We expect that high precision VLBI astrometry at the level of several micro-arcseconds will be achieved by global mm-VLBI radio telescopes having the simultaneous multi-frequency receiver system. The successful demonstrations with international partners (e.g., VERA, ATCA and Yebes) represent an important step towards this goal. At present, plausible options to provide $\mathrm{KVN}$-compatible system seem to have either a retrofitted KVN QO system (e.g., VERA and Yebes) or a triple feed-single dewar system. Fuelled by the interest in the phased-up ALMA, there is now a push to develop the simultaneous multi-frequency capability as part of the European VLBI Network (EVN).

Over the next couple of years, we expect that $\mathrm{x}-\mathrm{KVN}$ will be able to conduct regular observations of selected AGN samples to measure their core shifts at millimeter wavelengths and provides useful information to understand the physical structure and parameters of AGN jets and supermassive black holes. Lastly, it is worth noting that the core shift study can provide an important basis for the alignment between radio and optical reference frames by VLBI (ICRF: International Celes- tial Reference Frame) and the ESA space astrometry mission GAIA, respectively (Bourda et al. 2008).

\section{ACKNOWLEDGMents}

We acknowledge the crucial support of Korea Astronomy and Space Science Institute (KASI) [project name: A study on the core shift effect in AGN jets] and DFAT Australia-Korea Fund (GrantNo AKF2014-00010) in delivering the goals of this project. We are also grateful to the KREONET (Korea Research Environment Open NETwork), which is managed and operated by KISTI (Korea Institute of Science and Technology Information), for supporting KVN operations.

\section{REFERENCES}

Blandford, R. D., \& Königl, A. 1979, Relativistic Jets as Compact Radio Sources, ApJ, 232, 34

Bourda, G., Charlot, P., \& Le Campion, J.-F. 2008, Astrometric Suitability of Optically-Bright ICRF Sources for the Alignment with the Future Gaia Celestial Reference Frame, A\&A, 490, 403

Chi, S., Barthel, P. D., \& Garrett, M. A. 2013, Deep, Widefield, Global VLBI Observations of the Hubble Deep Field 
North (HDF-N) and Flanking Fields (HFF), A\&A, 550, A68

Dodson, R., \& Rioja, M. J. 2009, VLBA Scientific Memorandum n. 31: Astrometric Calibration of Mm-VLBI using "Source/Frequency Phase Referenced" Observations, Tech. Rept. NRAO.

Doeleman, S. S., Weintroub, J., Rogers, A. E. E., et al. 2008, Event-Horizon-Scale Structure in the Supermassive Black Hole Candidate at the Galactic Centre, Nature, 455, 78

Fomalont, E., Impellizzeri, V., \& Wilson, C. 2014, Technical Description and Implementation of Band to Band Phase Transfer, Tech. Rept. NRAO.

Garrett, M. A. 2005, Deep Field Surveys - A Radio View, eds. Gurvits, L. I., Frey, S., \& Rawlings, S., EAS Publications Series, 15, 73

Goldsmith, P. F. 1998, Quasioptical Systems: Gaussian Beam Quasioptical Propagation and Applications (New York: IEEE press)

Hada, K., Doi, A., Kino, M., et al. 2011, An Origin of the Radio Jet in M87 at the Location of the Central Black Hole, Nature, 477, 185

Han, S.-T., Lee, J.-W., Kang, J., et al. 2008, MillimeterWave Receiver Optics for Korean VLBI Network, Int. J. of Infrared and Millimeter Waves, 29, 69

Han, S.-T., Lee, J.-W., Kang, J., et al. 2013, Korean VLBI Network Receiver Optics for Simultaneous Multifrequency Observation: Evaluation, PASP, 125, 539

Hovatta, T., Aller, M. F., Aller, H. D., et al. 2014, MOJAVE: Monitoring of Jets in Active Galactic Nuclei with VLBA Experiments. XI. Spectral Distributions, AJ, 147, 143

Jung, T. 2011, A Study of KVN Multi-Frequency Phase Referencing, $\mathrm{PhD}$ dissertation, University of Science and Technology, Korea

Jung, T., Sohn, B. W., Kobayashi, H., et al. 2011, First Simultaneous Dual-Frequency Phase Referencing VLBI Observation with VERA, PASJ, 63, 375

Kim, H.-G., Han, S.-T., Sohn, B. W., et al. 2004, Construction of the Korean VLBI Network (KVN), eds. Bachiller, R., Colomer, F., Desmurs, J.-F., \& de Vicente, P., European VLBI Network on New Developments in VLBI Science and Technology, 281

Kovalev, Y. Y., Lobanov, A. P., Pushkarev, A. B., \& Zensus, J. A. 2008, Opacity in Compact Extragalactic Radio Sources and its Effect on Astrophysical and Astrometric Studies, A\&A, 483, 759

Krichbaum, T. P., Roy, A., Lu, R.-S., et al. 2014, Millimiter VLBI observations: Black Hole Physics and the Origin of Jets, Proceedings of the 12th European VLBI Network Symposium and Users Meeting (EVN 2014), 13

Lee, S.-S., Petrov, L., Byun, D.-Y., et al. 2014, Early Science with the Korean VLBI Network: Evaluation of System Performance, AJ, 147, 77

López-Pérez, J. A., Tercero, F., Serna, J. M., \& LópezFernández, J. A. 2012, A Tri-band Cryogenic Receiver for the RAEGE Project Antennas, eds. Behrend, D., \& Baver, K. D., Seventh General Meeting (GM2012) of the International VLBI Service for Geodesy and Astrometry (IVS), 66

Matthews, L., \& Crew, G. 2015, Summary of the First ALMA Phasing Project (APP) Commissioning and Science Verification Mission, Tech. Rept.

Middelberg, E., Roy, A. L, Walker, R. C., \& Falcke, H. 2005, VLBI Observations of Weak Sources Using Fast Frequency Switching, A\&A, 433, 897

Napier, P. J. 1995, VLBA Design, eds. Zensus, J. A., Diamond, P. J., \& Napier, P. J., Very Long Baseline Interferometry and the VLBA, ASP, 82, 59

Porcas, R. W., \& Rioja, M. J. 2002, VLBI Phase-Reference Investigations at $86 \mathrm{GHz}$, eds. Ros, E., Porcas, R. W., Lobanov, A. P., \& Zensus, J. A., Proceedings of the 6th EVN Symposium, 65

Reid, M. J., \& Honma, M. 2014, Microarcsecond Radio Astrometry, ARAA, 52, 339

Rioja, M., \& Dodson, R. 2011, High-Precision Astrometric Millimeter Very Long Baseline Interferometry Using a New Method for Atmospheric Calibration, AJ, 141, 114

Rioja, M., Dodson, R., Malarecki, J., \& Asaki, Y. 2011, Exploration of Source Frequency Phase Referencing Techniques for Astrometry and Observations of Weak Sources with High Frequency Space Very Long Baseline Interferometry, AJ, 142, 157

Rioja, M. J., Dodson, R., Jung, T., et al. 2014, Verification of the Astrometric Performance of the Korean VLBI Network, Using Comparative SFPR Studies with the VLBA at $14 / 7 \mathrm{~mm}$, AJ, 148, 84

Rioja, M. J., Dodson, R., Jung, T., \& Sohn, B. W. 2015, The Power of Simultaneous Multi-Frequency Observations for mm-VLBI: Astrometry up to $130 \mathrm{GHz}$ with the KVN, AJ, submitted

Sokolovsky, K. V., Kovalev, Y. Y., Pushkarev, A. B., \& Lobanov, A. P. 2011, A VLBA Survey of the Core Shift Effect in AGN Jets: I. Evidence of Dominating Synchrotron Opacity. A\&A, 532, A38

Williams, W., Nixon, D., Reilly, H., et al. 1979, A Prototype DSN XS Band Feed: DSS 13 Application Status (Third Report), DSN Progress Report 42, 51

Zorzi, P., Granet, C., Colleoni, F., et al. 2012, Construction and Measurement of a 31.3-45 GHz Optimized Splineprofile Horn with Corrugations, J. of Infrared, Millimeter, and Terahertz Waves, 33, 17 